\title{
Phenylureas CPPU and Thidiazuron Affect Yield Components, Fruit Composition, and Storage Potential of Four Seedless Grape Selections
}

\author{
A.G. Reynolds ${ }^{1}$, D.A. Wardle ${ }^{2}$, C. Zurowski ${ }^{2}$, and N.E. Looney ${ }^{1}$ \\ Agriculture Canada Research Station, Summer-land, B.C. VOH 1Z0 Canada
}

Additional index word. growth regulators, cytokinins, table grapes, Vitis

\begin{abstract}
One of three levels $\left(0,1,10 \mathrm{mg}^{-1 i t e r}{ }^{-1}\right)$ of the cytokinin-active substituted phenylurea compound CPPU was applied with or without $100 \mathrm{mg} \mathrm{GA} /$ liter to developing clusters of 'Sovereign Coronation' and Summerland Selection 495 grapes (Vitis spp.). In a similar experiment, one of three levels $\left(0,1,10 \mathrm{mg} \cdot \mathrm{liter}^{-1}\right)$ of either CPPU or the related compound thidiazuron was applied to 'Simone' and Summerland Selection 535. Both phenylurea chemicals tended to linearly increase cluster weight and berry weight while reducing degrees Brix, pH, and anthocyanins and increasing titratable acidity. A subsequent trial with 0,4 , and $8 \mathrm{mg}$ thidiazuron/liter on all four varieties yielded similar results. GA had no individual or synergistic effects. Due to the very low concentrations required, CPPU and thidiazuron show great promise as chemical tools for the increase of berry weight in seedless table grapes. Chemical names used: $\mathrm{N}$-(2-chloro-4-pyridyl) - $\mathrm{N}^{\prime}$-phenylurea (CPPU); $\mathrm{N}^{1}$-phenyl-N'-1,2,3- thiadiazol-5-yl urea (thidiazuron);
\end{abstract}

Berry size and cluster conformation of seedless table grapes are customarily improved through the application of growth regulators. Growers of 'Thompson Seedless' in California apply as much as $40 \mathrm{mg} \mathrm{GA} /$ liter during each of two annual applications to thin berries and increase berry size. One result of public pressure to reduce agricultural chemical use has been a renewed interest in finding even more powerful growth regulators that can be used at very low concentrations months ahead of harvest to eliminate product residues. Another strategy is to target specific plant organs with the chemical to reduce the amount of chemical applied per hectare.

The cytokinins are plant growth regulators with the ability to enhance plant cell division and cell expansion, as well as delay senescence. However, their use in viticulture has been relatively limited. Weaver et al. (1966) found that cluster dips of benzyladenine and 6-(benzylamino)-9-(2-tetra-hydropyranyl)-9H-purine (BTP) at 500 to $1000 \mathrm{mg} \cdot$ liter $^{-1}$ led to increased berry set and size, but with some reduction in degrees Brix, in several varieties.

More recently, interest has been shown in the substituted phenylurea compounds in terms of their activity on grapes and tree fruits. Nickell (1985) first reported on the benefits of CPPU cluster dips on 'Thompson Seedless' berry weight. He found that 2.5 to $10.0 \mathrm{mg} \cdot$ liter $^{-1}$ cluster dips were effective, but that full-vine sprays at these rates achieved only $\approx 60 \%$ of the response obtained from the cluster dips. These results were later confirmed with 'Flame' (Nickell, 1986a) and 'Perlette' (Nickell, 1986b). A related compound, CN-11-3183, has been shown by Morris et al. (1986) to increase berry size of 'Concord' and 'Reliance' (V. labruscana L.). The beneficial effects of both CPPU and CN-11-3183 were enhanced by GA (Morris et al. 1986; Nickell, 1985). Fruit size increases resulting from CPPU have also been reported for other fruits (Iwahori et al., 1988; Nickell, 1986b; Ogata et al., 1988).

Seedless table grapes are a relatively new and expanding in-

Received for publication 19 Mar. 1991. Accepted for publication 10 Sept. 1991. Contribution no. 779. The cost of publishing this paper was defrayed in part by the payment of page charges. Under postal regulations, this paper therefore must be hereby marked advertisement solely to indicate this fact.

${ }^{\mathrm{I}}$ Research Scientist.

${ }^{2}$ Research Assistant. dustry in British Columbia. The marketability of the crop will partially depend on appearance, especially berry size. The purpose of these investigations was to test the effectiveness of CPPU, the synergistic effects of GA, if any, and the comparative effects of CPPU and thidiazuron on yield components, fruit composition, and storage potential of four seedless table grape selections and cultivars. For purposes of clarity, the term "varieties" will be used throughout this paper to refer to either "cultivars" or "selections."

\section{Materials and Methods}

CPPU and gibberellic acid (Expt. 1). Five vines each of 'Sovereign Coronation' (blue-skinned) (Denby, 1977) and Summerland Selection 495 were selected in May 1989 for the study of the effects of CPPU and the potential synergistic effects with GA. 'Sovereign Coronation' vines were spaced 1.8 x $3.3 \mathrm{~m}$ (vine $\mathrm{x}$ row) and were trained to a divided canopy system borne on a Y-shaped trellis, with cordon wires at $1.3 \mathrm{~m}$, and with a 0.3-m horizontal separation at the cordon level. Postbloom upward vertical shoot positioning maintained the divided canopy that was hedged $0.9 \mathrm{~m}$ above the cordon wire. Subcanopies were horizontally separated at the top by $1.6 \mathrm{~m}$. Selection 495 vines were spaced $1.8 \times 3.3 \mathrm{~m}$ and were trained to a $1.8-\mathrm{m}$ high bilateral cordon. Both varieties were pruned to three-node spurs and shoots were later adjusted to 30 shoots per vine or subcanopy. Pest control and soil management practices were carried out in accordance with published recommendations (B.C. Min. Agr. and Fisheries, 1987).

Each subcanopy ('Sovereign Coronation') or vine (Selection 495) was divided into six equal sections in anticipation of treatment application. Within each section, two typical shoots were chosen and flagged, and were cluster-thinned to one cluster per shoot. One of three concentrations of CPPU $\left(0,1,10 \mathrm{mg} \cdot\right.$ liter $\left.^{-1}\right)$ with or without $100 \mathrm{mg} \mathrm{GA} / \mathrm{liter}$ (six treatment combinations total) was assigned randomly to one of the six pairs of shoots on each vine or subcanopy. Thus, the design was a randomized complete block, with individual vines as blocks, and the groups of two (Selection 495) or four ('Sovereign Coronation') shoots bearing the treated clusters as treatment replicates within the blocks. Representative clusters were given a 1- to 3-see dip. Treatments were applied on 13 July 1989 when berries were 5 to $6 \mathrm{~mm}$ in diameter. All treatment solutions contained $1 \mathrm{ml}$ 
Triton surfactant/liter and $42 \mathrm{ml} 95 \%$ ethanol/liter to dissolve the CPPU.

Individual clusters were harvested on 12 Sept. ('Sovereign Coronation') and 19 Sept. (Selection 495). Two representative clusters per vine or subcanopy were also sampled from non-cluster-thinned shoots. One of each pair of clusters per vine from each treatment combination on Selection 495, and two of the four clusters per vine from each treatment combination on 'Sovereign Coronation' were used immediately for analysis of yield components and fruit composition. The remaining clusters were weighed, and subsequently stored at $2 \mathrm{C}$. The largest clusters within each treatment replicate were retained for storage.

Cluster weight, berry weight, number of berries, and rachis weight and length were recorded for unstored fruit. Berries were then juiced, and degrees Brix, $\mathrm{pH}$, and titratable acidity (TA) were determined on settled juice using an Abbé refractometer (AO Scientific, Buffalo, N.Y.), a Fisher 825 MP digital pH meter (Fisher Scientific, Vancouver, B.C.), and a Metrohmn 672 Titroprocessor (Brinkman Instruments Canada, Rexdale, Ont.), respectively. Anthocyanins in the 'Sovereign Coronation' juice were determined by the method of Métivier et al. (1980) using malvidin-3,5-diglucoside as a standard. Anthocyanin levels and juice absorbance values (OD420 and OD520) were measured on a Gilford Response UV-VIS spectrophotometer (Ciba-Corning Canada, Richmond Hill, Ont.). Tristimulus values (L, a, b) of the reflected skin color of 'Sovereign Coronation' were measured on five berries selected at random from each cluster using a Minolta CR-200 Chroma Meter (Minolta Camera, Japan).

Stored clusters were removed from storage on 11 and 12 Dec. 1989. All yield component and fruit composition measurements made on the fresh fruit were likewise carried out on the stored samples. Determinations were also made on a per-cluster basis on: moldy berries, shriveled berries, shattered berries upon removal from storage and after $48 \mathrm{~h}$ at 20C, and rachis drying.

CPPU and thidiazuron (Expt. 2). Five vines each of 'Simone' (Reynolds et al., 1989) and Summerland Selection 535 were chosen to investigate comparative effects of CPPU and thidiazuron. Training, pruning, and spacing of 'Simone' were identical to 'Sovereign Coronation', while all cultural aspects for Selection 535 were the same as for Selection 495.

Each subcanopy ('Simone') or vine (Selection 535) was divided into six sections. Two shoots per section were flagged and cluster-thinned, and one of three levels of CPPU $(0,1,10$ mg.liter $\left.{ }^{-1}\right)$ or thidiazuron $\left(0,1,10 \mathrm{mg} \cdot\right.$ liter $\left.^{-1}\right)$ was applied on
13 July 1989 to one of the six pairs of shoots on subcanopy or vine, as described under Expt. 1. Berries were 5 to $6 \mathrm{~mm}$ in diameter at the time of treatment. A randomized complete-block design was used with five blocks (vines) and six treatments (groups of shoots). Clusters were given a 1- to 3-sec dip. Solutions of CPPU and thidiazuron contained $1 \mathrm{ml}$ Triton surfactant/liter and $42 \mathrm{ml} \mathrm{95 \%} \mathrm{ethanol/liter.}$

Individual clusters of 'Simone' were harvested 28 Sept. 1989 and Selection 535 on 12 Sept. Determinations of yield components and fruit composition of 'Simone' and Selection 535 were as described under Expt. 1 for 'Sovereign Coronation' and Selection 495, respectively. Stored fruit was removed from storage on 11-12 Dec. 1989 and analyzed as described for Expt. 1. The largest clusters in each treatment replicate again had been retained for storage.

Intermediate concentrations of thidiazuron. (Expt. 3). The vines of the four selections used in 1989 were used in 1990 to test the effectiveness of intermediate levels of thidiazuron. All cultural practices were as in 1989. Each subcanopy ('Sovereign Coronation' and 'Simone') or vine (Selections 495 and 535) was divided into three sections in anticipation of treatment application, and two shoots per section were flagged and clusterthinned to one cluster per shoot. The design was a randomized complete block with five blocks (vines) and three treatments (groups of shoots). Thidiazuron at 0,4 , or $8 \mathrm{mg} \cdot$ liter $^{-1}$ was applied on 23 July 1990 when the berry diameter was 9 to 12 $\mathrm{mm}$. All solutions contained $1 \mathrm{mg}$ Triton surfactant/liter and 42 $\mathrm{ml} \mathrm{95 \%} \mathrm{ethanol/liter.}$

Individual clusters were harvested on 1 Oct. 1990 ('Sovereign Coronation' and 'Simone') and 17 Sept. 1990 (Selections 495 and 535), Determinations of yield components and fruit composition were the same as in 1989, except no measurements of color components (juice anthocyanins; juice absorbance; berry skin L, a, b) were carried out on 'Sovereign Coronation' and 'Simone'. Clusters destined for storage were placed on $\mathrm{SO}_{2}$ generating pads within fiberboard boxes that were double-wrapped with $0.05 \mathrm{~mm}$ polyethylene. As in Expts. 1 and 2, the largest clusters in each treatment replicate were those designated for storage. Storage was at 2C. Clusters were removed from storage on 10 Dec. 1990 and determinations of yield components, fruit composition, and post-storage condition were made as in Expts. 1 and 2.

All data were subjected to analysis of variance using the GLM procedure of the SAS statistical package (SAS Institute, Cary, N.C.).

Table 1. Effects of CPPU concentration on yield components and fruit composition of 'Sovereign Coronation' and Selection 495 grapes, 1989.

\begin{tabular}{|c|c|c|c|c|c|c|c|c|c|c|c|c|c|c|}
\hline \multirow{2}{*}{$\begin{array}{l}\text { CPPU } \\
\text { (mg-liter-1) }\end{array}$} & \multicolumn{2}{|c|}{$\begin{array}{l}\text { Rachis } \\
\text { length } \\
(\mathrm{mm})\end{array}$} & \multicolumn{2}{|c|}{$\begin{array}{c}\text { Cluster } \\
\text { wt } \\
(\mathrm{g})\end{array}$} & \multicolumn{2}{|c|}{$\begin{array}{c}\text { Berry } \\
\text { wt } \\
(\mathrm{g})\end{array}$} & \multicolumn{2}{|c|}{$\begin{array}{c}\text { Berries/ } \\
\text { cluster }\end{array}$} & \multicolumn{2}{|c|}{${ }^{\circ} \mathrm{Brix}$} & \multicolumn{2}{|c|}{$\begin{array}{c}\text { Titratable } \\
\text { acidity } \\
\left(\mathrm{g} \cdot \text { liter }^{-1}\right)\end{array}$} & \multicolumn{2}{|c|}{$\mathrm{pH}$} \\
\hline & $F^{2}$ & $S^{z}$ & $\mathrm{~F}$ & $\mathbf{S}$ & $\mathrm{F}$ & $\mathbf{S}$ & $\mathbf{F}$ & $S$ & $\mathrm{~F}$ & $S$ & $\mathrm{~F}$ & $\mathrm{~S}$ & $\mathrm{~F}$ & $S$ \\
\hline 0 & 130 & 141 & 136.6 & 214.1 & 1.92 & 1.57 & 67.2 & 104.8 & 20.6 & 22.2 & 10.7 & 13.6 & 3.08 & 2.97 \\
\hline 1 & 133 & 150 & 161.9 & 249.7 & 2.21 & 2.10 & 70.4 & 97.8 & 19.9 & 21.6 & 11.5 & 13.6 & 3.06 & 2.93 \\
\hline 10 & 134 & 146 & 208.2 & 339.8 & 2.88 & 2.51 & 69.0 & 110.1 & 18.6 & 19.7 & 12.1 & 13.8 & 3.02 & 2.90 \\
\hline 0 & 177 & 159 & 191.9 & 244.9 & 2.50 & 1.90 & 73.0 & 85.4 & 17.5 & 25.0 & 14.9 & 15.0 & 2.76 & 2.83 \\
\hline 1 & 183 & 187 & 226.6 & 339.8 & 2.46 & 2.09 & 85.5 & 108.5 & 18.6 & 24.3 & 14.1 & 14.6 & 2.79 & 2.85 \\
\hline 10 & 197 & 201 & 386.0 & 458.4 & 3.13 & 2.61 & 127.0 & 128.8 & 17.0 & 22.4 & 15.3 & 14.9 & 2.76 & 2.84 \\
\hline Significance & NS & ${ }^{*} \mathrm{~L}$ & ${ }^{* * *} \mathrm{~L} ;{ }^{*} \mathrm{Q}$ & $* * * L$ & ${ }^{* *} \mathrm{~L}$ & ${ }^{* * *} \mathrm{~L}$ & ${ }^{* *} \mathrm{~L}$ & ${ }^{*} \mathrm{~L}$ & ${ }^{*} \mathrm{Q}$ & ${ }^{* *} \mathrm{~L}$ & NS & NS & NS & NS \\
\hline
\end{tabular}

${ }^{2} \mathrm{~F}=$ Fresh; $\mathrm{S}=$ stored.

$\mathrm{NS}, *, * *, * * *$ Nonsignificant or significant at $\mathrm{R} \quad 0.05,0.01$, or 0.001 , respectively. $\mathrm{L}=$ linear, $\mathrm{Q}=$ quadratic. 


\begin{tabular}{|c|c|c|c|c|c|c|c|c|c|c|c|c|c|c|c|c|}
\hline \multirow{3}{*}{$\begin{array}{c}\text { CPPU } \\
\text { (mg.liter-1) }\end{array}$} & \multicolumn{6}{|c|}{ Tristimulus color } & \multicolumn{8}{|c|}{ Absorbance } & \multirow{2}{*}{\multicolumn{2}{|c|}{$\begin{array}{c}\text { Total } \\
\text { anthocyanins } \\
\left(\mathrm{mg} \cdot \text { liter }^{-1}\right)\end{array}$}} \\
\hline & \multicolumn{2}{|c|}{$\mathrm{L}$} & \multicolumn{2}{|c|}{$-\mathrm{a}$} & \multicolumn{2}{|c|}{$-b$} & \multicolumn{2}{|c|}{ OD420 } & \multicolumn{2}{|c|}{ OD520 } & \multicolumn{2}{|c|}{ Hue $^{y}$} & \multicolumn{2}{|c|}{ Intensity ${ }^{\mathbf{x}}$} & & \\
\hline & $\mathrm{F}^{\mathrm{z}}$ & $S^{\mathbf{z}}$ & $\mathrm{F}$ & $S$ & $\mathrm{~F}$ & $\mathrm{~S}$ & $\mathrm{~F}$ & $\mathbf{S}$ & $\mathrm{F}$ & $\mathrm{S}$ & $\mathrm{F}$ & $\mathrm{S}$ & $\mathrm{F}$ & $\mathrm{S}$ & $\mathrm{F}$ & $S$ \\
\hline 0 & 26.62 & 29.92 & 1.32 & 1.65 & 6.74 & 5.18 & 0.80 & 0.21 & 0.74 & 0.39 & 1.20 & 0.61 & 1.54 & 0.60 & 19.2 & 163.5 \\
\hline 1 & 26.22 & 29.64 & 1.50 & 1.76 & 7.05 & 5.16 & 0.71 & 0.17 & 0.76 & 0.32 & 1.00 & 0.59 & 1.48 & 0.49 & 20.6 & 131.5 \\
\hline 10 & 26.97 & 29.48 & 2.12 & 2.29 & 6.92 & 5.02 & 0.46 & 0.10 & 0.41 & 0.15 & 1.17 & 0.66 & 0.88 & 0.25 & 6.8 & 48.3 \\
\hline Significance & NS & NS & $* * * \mathrm{~L}$ & ${ }^{* * *} \mathrm{~L}$ & NS & NS & $* * * \mathrm{~L}$ & $* * * \mathrm{~L}$ & ${ }^{* * *} \mathrm{~L}$ & ${ }^{* * *} \mathrm{~L}$ & NS & ${ }^{* *} \mathrm{~L}$ & $* * * \mathrm{~L}, \mathrm{Q}$ & $* * * \mathrm{~L}$ & ${ }^{* *} \mathrm{~L}$ & $* * * \mathrm{~L}$ \\
\hline
\end{tabular}

${ }^{2} \mathrm{~F}=$ Fresh; $\mathrm{S}=$ stored.

${ }^{y}$ Hue $=$ OD420/OD520.

${ }^{x}$ Intensity $=$ OD420 + OD520.

$\mathrm{NS}, * *, * * *$ Nonsignificant or significant at $\mathrm{P} \leq 10.01$ or 0.001 , respectively. $\mathrm{L}=$ linear, $\mathrm{Q}=$ quadratic.

Table3. Effects of CPPU and thidiazuron concentration on yield components and fruit composition of Selection 535 and 'Simone' grapes, 1989.

\begin{tabular}{|c|c|c|c|c|c|c|c|c|c|c|}
\hline \multirow{2}{*}{$\begin{array}{c}\text { Chemical } \\
\left(\mathrm{mg} \cdot \text { liter }^{-1}\right)\end{array}$} & \multicolumn{2}{|c|}{$\begin{array}{l}\text { Cluster wt } \\
\text { (g) }\end{array}$} & \multicolumn{2}{|c|}{$\begin{array}{l}\text { Berry } \\
\text { wt (g) }\end{array}$} & \multicolumn{2}{|c|}{${ }^{\circ}$ Brix } & \multicolumn{2}{|c|}{$\begin{array}{l}\text { Titratable } \\
\text { acidity } \\
\left(\mathrm{g} \cdot \mathrm{liter}^{-1}\right)\end{array}$} & \multicolumn{2}{|c|}{$\mathrm{pH}$} \\
\hline & $F^{2}$ & $S^{z}$ & $\mathrm{~F}$ & $S$ & $\mathbf{F}$ & $S$ & $F$ & $S$ & $\mathrm{~F}$ & $S$ \\
\hline & \multicolumn{10}{|c|}{ Simone } \\
\hline CPPU & & & & & & & & & & \\
\hline 0 & 104.0 & 162.6 & 1.42 & 1.22 & 19.8 & 24.0 & 8.1 & 6.9 & 3.09 & 3.24 \\
\hline 1 & 119.3 & 203.2 & 1.46 & 1.34 & 20.4 & 23.1 & 8.1 & 7.4 & 3.11 & 3.22 \\
\hline 10 & 166.8 & 234.3 & 1.78 & 1.47 & 19.9 & 23.1 & 8.1 & 7.3 & 3.13 & 3.22 \\
\hline Significance & $* * \mathrm{~L}$ & $* * * \mathrm{~L}$ & $* * \mathrm{~L}$ & ${ }^{*} \mathrm{~L}$ & NS & NS & NS & ${ }^{*} \mathrm{Q}$ & NS & NS \\
\hline \multicolumn{11}{|l|}{ Thidiazuron } \\
\hline 0 & 85.6 & 140.6 & 1.34 & 1.12 & 20.8 & 24.6 & 7.6 & 7.2 & 3.14 & 3.23 \\
\hline 1 & 101.4 & 180.9 & 1.47 & 1.24 & 20.8 & 23.4 & 8.0 & 7.4 & 3.14 & 3.23 \\
\hline 10 & 119.8 & 240.5 & 1.77 & 1.56 & 20.2 & 23.4 & 8.1 & 7.2 & 3.11 & 3.23 \\
\hline Significance & NS & ${ }^{* * *} \mathrm{~L}$ & $* * * \mathrm{~L}$ & $* * * \mathrm{~L}$ & NS & NS & ${ }^{*} \mathrm{~L}$ & NS & ${ }^{*} \mathrm{~L}$ & NS \\
\hline CPPU & \multicolumn{10}{|c|}{ Selection 535} \\
\hline 0 & 195.7 & 246.6 & 1.70 & 1.29 & 24.4 & 29.5 & 13.6 & 14.7 & 3.00 & 2.99 \\
\hline 1 & 201.6 & 323.0 & 1.91 & 1.65 & 22.7 & 29.8 & 15.3 & 14.2 & 2.97 & 3.04 \\
\hline 10 & 252.7 & 375.0 & 2.09 & 1.56 & 21.6 & 28.0 & 15.2 & 16.4 & 2.92 & 2.95 \\
\hline Significance & NS & NS & NS & NS & ${ }^{* *} \mathrm{~L}$ & NS & ${ }^{* *} \mathrm{~L}$ & NS & NS & NS \\
\hline Thidiazuron & & & & & & & & & & \\
\hline 0 & 149.4 & 214.4 & 1.59 & 1.14 & 23.4 & 31.7 & 14.8 & 15.7 & 2.95 & 2.96 \\
\hline 1 & 230.9 & 281.8 & 1.74 & 1.32 & 22.9 & 31.0 & 14.2 & 16.5 & 2.93 & 2.90 \\
\hline 10 & 324.6 & 452.3 & 2.40 & 1.94 & 21.8 & 27.0 & 15.8 & 17.0 & 2.94 & 2.96 \\
\hline Significance & $* * \mathrm{~L}$ & ${ }^{*} \mathrm{~L}$ & $* * * \mathrm{~L}$ & ${ }^{* *} \mathrm{~L}$ & NS & $* * * \mathrm{~L}$ & ${ }^{*} \mathrm{Q}$ & NS & NS & NS \\
\hline
\end{tabular}

\section{Results}

Expt. 1. Cluster weights and berry weights of 'Sovereign Coronation' and Selection 495 increased linearly with increasing CPPU concentration (Table 1). Berries per cluster and rachis length (stored clusters only) in Selection 495 also increased linearly with CPPU concentration. CPPU tended to delay fruit maturity, indicated by a linear reduction in degrees Brix for both selections, as well as by an increase in TA and a decrease in $\mathrm{pH}$ for 'Sovereign Coronation'.

Some values for juice and berry color in 'Sovereign Coronation' were also reduced by CPPU; OD420 and OD520, intensity, and total anthocyanins were reduced linearly with increasing CPPU level (Table 2). The 'a' component of the tristimulus color reading decreased with CPPU level, indicating a linear decrease in the red color component. Skins of berries treated with 1 or $10 \mathrm{mg}$ CPPU/liter were noticeably more red and less blue than those treated with $0 \mathrm{mg} \cdot$ liter -1 The increase in hue in the juice of stored clusters with CPPU level suggests that the proportion of red : brown pigments in the juice decreased with increasing CPPU level.

Effects of GA were generally not significant (data not shown). A significant CPPU $\times$ GA interaction in the two varieties for rachis length of stored clusters suggested that GA could improve the tendency for CPPU to increase rachis length (data not shown). GA also increased the ' $a$ ' component of the tristimulus color reading in stored clusters of 'Sovereign Coronation' from - 2.03 to -1.77 , suggesting that the skins of GA-treated 'Sovereign Coronation' berries were more red and less green than those not treated with GA (data not shown).

There were no apparent effects of CPPU or GA on poststorage condition of either variety (data not shown). Berries of both varieties showed slight to moderate signs of turgor loss 


\begin{tabular}{|c|c|c|c|c|c|c|c|c|c|c|c|c|c|c|c|c|}
\hline \multirow{2}{*}{$\begin{array}{c}\text { Chemical } \\
\left(\mathrm{mg} \cdot \text { liter }^{-1}\right)\end{array}$} & \multicolumn{6}{|c|}{ Tristimulus color } & \multicolumn{8}{|c|}{ Absorbance } & \multicolumn{2}{|c|}{$\begin{array}{c}\text { Total } \\
\text { anthocyanins } \\
\left(\mathrm{mg} \cdot \text { liter }^{-1}\right)\end{array}$} \\
\hline & $\mathrm{F}^{\mathbf{z}}$ & $S^{\mathbf{z}}$ & $\mathrm{F}$ & $\mathbf{S}$ & $\mathrm{F}$ & $\mathbf{S}$ & $\mathrm{F}$ & $S$ & $\mathrm{~F}$ & $S$ & $\mathrm{~F}$ & $S$ & F & $S$ & $\mathrm{~F}$ & $\mathrm{~S}$ \\
\hline 0 & 30.99 & 29.99 & 2.07 & 2.24 & 5.16 & 5.03 & 0.47 & 0.81 & 0.66 & 0.99 & 0.75 & 0.90 & 1.13 & 1.80 & 42.8 & 42.3 \\
\hline 1 & 30.79 & 29.61 & 2.09 & 2.38 & 5.12 & 4.97 & 0.44 & 0.66 & 0.67 & 0.78 & 0.70 & 0.87 & 1.11 & 1.44 & 45.4 & 29.5 \\
\hline 10 & 31.45 & 30.56 & 2.39 & 2.88 & 5.33 & 5.29 & 0.37 & 0.63 & 0.54 & 0.61 & 0.75 & 1.08 & 0.91 & 1.24 & 34.2 & 19.6 \\
\hline $\begin{array}{c}\text { Thidi } \\
0\end{array}$ & 31 & 2957 & 197 & 225 & 5 & & & & & & & & & & & \\
\hline 1 & 32.31 & 28.81 & 2.03 & 2.82 & 5.03 & 4.20 & 0.43 & $\begin{array}{l}0.10 \\
0.63\end{array}$ & $\begin{array}{l}0.02 \\
0.61\end{array}$ & $\begin{array}{l}0.92 \\
0.78\end{array}$ & $\begin{array}{l}0.02 \\
0.72\end{array}$ & $\begin{array}{l}0.04 \\
0.84\end{array}$ & $\begin{array}{l}1.12 \\
1.04\end{array}$ & $\begin{array}{l}1.00 \\
.1 .41\end{array}$ & $\begin{array}{l}40.1 \\
37.4\end{array}$ & $\begin{array}{l}50.0 \\
30.7\end{array}$ \\
\hline 10 & 32.61 & 28.78 & 2.29 & 3.11 & 5.41 & 4.35 & 0.37 & 0.56 & 0.51 & 0.62 & 0.78 & 0.93 & 0.89 & 1.17 & 28.7 & 21.8 \\
\hline Significance & NS & NS & NS & ${ }^{* *} \mathrm{~L}$ & NS & NS & ${ }^{* *} \mathrm{~L}$ & ${ }^{* *} \mathrm{~L}$ & NS & ${ }^{* *} \mathrm{~L}$ & NS & NS & ${ }^{* *} \mathrm{~L}$ & ${ }^{* *} \mathrm{~L}$ & ${ }^{*} \mathrm{~L}$ & ${ }^{* * *} \mathrm{~L}$ \\
\hline
\end{tabular}

${ }^{\mathrm{z}} \mathrm{F}=$ Fresh; $\mathrm{S}=$ stored.

${ }^{y}$ Hue $=$ OD420/OD520.

${ }^{x}$ Intensity $=$ OD420 + OD520

$\mathrm{NS}, *, * *, * *$ Nonsignificant or significant at $\underline{\underline{R}} 0.05,0.01$, or 0.001 , respectively. $\mathrm{L}=$ linear trend.

Table 5. Effects of thidiazuron concentration on yield components and fruit composition of four seedless grape varieties, 1990.

\begin{tabular}{|c|c|c|c|c|c|c|c|c|c|c|}
\hline \multirow{2}{*}{$\begin{array}{c}\text { Concn } \\
\left(\mathrm{mg} \cdot \text { liter }^{-1}\right)\end{array}$} & \multicolumn{2}{|c|}{$\begin{array}{c}\text { Cluster wt } \\
\text { (g) }\end{array}$} & \multicolumn{2}{|c|}{$\begin{array}{c}\text { Berry wt } \\
(\mathrm{g})\end{array}$} & \multicolumn{2}{|c|}{${ }^{\circ}$ Brix } & \multicolumn{2}{|c|}{$\begin{array}{l}\text { Titratable } \\
\text { acidity } \\
\left(\mathrm{g} \cdot \text { liter }^{-1}\right)\end{array}$} & \multicolumn{2}{|c|}{$\mathrm{pH}$} \\
\hline & $\mathrm{F}^{\mathbf{z}}$ & $S^{\mathbf{z}}$ & $\mathrm{F}$ & $\mathbf{S}$ & $\mathbf{F}$ & $S$ & $\mathrm{~F}$ & $S$ & $\mathrm{~F}$ & $\mathbf{S}$ \\
\hline & \multicolumn{10}{|c|}{ Sovereign Coronation } \\
\hline 0 & 95.3 & 176.6 & 1.90 & 1.81 & 22.5 & 23.8 & 13.4 & 13.6 & 3.14 & 3.07 \\
\hline 4 & 88.3 & 173.0 & 2.07 & 1.95 & 22.3 & 23.5 & 13.1 & 12.8 & 3.14 & 3.07 \\
\hline 8 & 108.4 & 266.6 & 2.00 & 2.49 & 23.0 & 22.4 & 13.0 & 12.1 & 3.16 & 3.08 \\
\hline Significance & NS & ${ }^{*} \mathrm{~L}$ & NS & $* * * \mathrm{~L}$ & NS & ${ }^{* *} \mathrm{~L}$ & NS & $* * * \mathrm{~L}$ & NS & NS \\
\hline & \multicolumn{10}{|c|}{ Simone } \\
\hline 0 & 38.4 & 93.0 & 0.52 & 0.87 & 21.5 & 24.6 & 12.9 & 10.4 & 3.13 & 3.05 \\
\hline 4 & 77.4 & 163.7 & 0.87 & 1.58 & 21.9 & 21.8 & 11.9 & 8.9 & 3.14 & 3.10 \\
\hline 8 & 70.7 & 181.0 & 0.68 & 1.77 & 21.9 & 21.2 & 11.6 & 9.4 & 3.13 & 3.06 \\
\hline Significance & NS & ${ }^{*} \mathrm{~L}$ & ${ }^{*} \mathrm{Q}$ & NS & NS & $* * L$ & ${ }^{*} \mathrm{~L}$ & NS & ${ }^{*} Q$ & NS \\
\hline & \multicolumn{10}{|c|}{ Selection 495} \\
\hline 0 & 243.7 & 318.6 & 2.83 & 2.51 & 17.7 & 19.2 & 11.5 & 11.8 & 3.02 & 3.05 \\
\hline .4 & 331.2 & 509.2 & 3.34 & 3.20 & 16.0 & 17.1 & 14.6 & 13.3 & 2.90 & 2.97 \\
\hline 8 & 334.8 & 482.0 & 3.42 & 3.17 & 14.4 & 16.4 & 15.2 & 13.8 & 2.88 & 2.95 \\
\hline Significance & NS & ${ }^{*} \mathrm{~L}$ & ${ }^{*} \mathrm{~L}$ & ${ }^{*} \mathrm{~L}$ & ${ }^{* *} \mathrm{~L}$ & NS & ${ }^{* *} \mathrm{~L}$ & NS & ${ }^{* *} \mathrm{~L}$ & NS \\
\hline & \multicolumn{10}{|c|}{ Selection 535} \\
\hline 0 & 201.7 & 295.8 & 1.69 & 1.76 & 19.1 & 23.1 & 15.0 & 12.9 & 2.95 & 3.03 \\
\hline 4 & 313.1 & 416.1 & 2.66 & 2.38 & 18.1 & 19.1 & 16.4 & 15.1 & 2.91 & 2.93 \\
\hline 8 & 332.6 & 381.9 & 3.21 & 2.30 & 19.1 & 19.9 & 15.4 & 15.4 & 2.93 & 2.93 \\
\hline Significance & ${ }^{*} \mathrm{~L}$ & NS & NS & ${ }^{* *} \mathrm{~L}, \mathrm{Q}$ & NS & ${ }^{* *} \mathrm{~L},{ }^{*} \mathrm{Q}$ & NS & ${ }^{*} \mathrm{~L}$ & NS & ${ }^{*} \mathrm{~L}$ \\
\hline
\end{tabular}

and extensive rachis drying following $\approx 90$ clays of storage. Overall weight loss in storage averaged $20 \%$ for 'Sovereign Coronation' and $25 \%$ for Selection 495.

Expt. 2. CPPU had no significant effect on yield components of Selection 535, but increased cluster weight and berry weight of 'Simone' (Table 3). CPPU also decreased degrees Brix in Selection 535 and increased TA in both selections. Thidiazuron increased cluster weight, berry weight, and TA, of both varieties, decreased degrees Brix in Selection 535, and decreased $\mathrm{pH}$ in 'Simone' (Table 3).

Color components of 'Simone' were affected somewhat by both chemicals (Table 4). The 'a' component of the tristimulus color was decreased linearly with increasing concentrations of both chemicals. OD420 and OD520, intensity, and anthocyanins decreased linearly with increasing concentrations of both chemicals. Relative degrees of brown: red pigment in the juice of stored clusters appeared to increase proportional to level of CPPU, indicated by the hue reading.

There were no significant treatment differences on the poststorage condition of 'Simone' (data not shown). Overall, berries showed a moderate degree of turgor loss, extensive rachis desiccation, and considerable shatter after $48 \mathrm{~h}$ at 20C. Total weight loss in storage averaged $28 \%$ for 'Simone'. For Selection 535, thidiazuron at $10 \mathrm{mg} \cdot$ liter $^{-1}$ was associated with fewer shriveled berries than the control. The 0 and $1 \mathrm{mg} \cdot$ liter $^{-1}$ levels of thidiazuron displayed the most shattered berries of the six treatment 
Table 6. Effect of thidiazuron concentration on condition following storage of clusters of four seedless group varieties. 1990.

\begin{tabular}{|c|c|c|c|c|c|}
\hline Concn & $\begin{array}{l}\text { Cluster wt } \\
\text { after storage } \\
\text { (g) }\end{array}$ & $\begin{array}{l}\text { Wt loss } \\
\text { in storage } \\
(\mathrm{g})\end{array}$ & $\begin{array}{c}\text { Rachis } \\
\text { wt } \\
\text { (g) }\end{array}$ & $\begin{array}{c}\text { Shatter } \\
\text { at } \\
0 \mathrm{~h}^{\mathbf{z}}\end{array}$ & $\begin{array}{l}\text { Rachis } \\
\text { drying }\end{array}$ \\
\hline & \multicolumn{5}{|c|}{ Sovereign Coronation } \\
\hline 0 & 165.2 & 11.4 & 12.9 & 4.7 & 3.0 \\
\hline 4 & 156.7 & 16.4 & 12.8 & 2.9 & 2.9 \\
\hline 8 & 249.0 & 17.6 & 18.0 & 4.7 & 2.9 \\
\hline \multirow[t]{2}{*}{ Significance } & ${ }^{*} \mathrm{~L}$ & ${ }^{*} \mathrm{~L}$ & ${ }^{* *} \mathrm{~L}$ & NS & NS \\
\hline & \multicolumn{5}{|c|}{ Simone } \\
\hline 0 & 81.9 & 11.0 & 6.5 & 2.2 & 4.0 \\
\hline 4 & 149.3 & 14.4 & 8.6 & 3.8 & 3.6 \\
\hline 8 & 162.9 & 18.1 & 9.8 & 6.9 & 3.8 \\
\hline \multirow[t]{2}{*}{ Significance } & ${ }^{*} \mathrm{~L}$ & ${ }^{*} \mathrm{~L}$ & ${ }^{*} \mathrm{~L}$ & ${ }^{* *} \mathrm{~L}$ & NS \\
\hline & \multicolumn{5}{|c|}{ Selection 495} \\
\hline 0 & 282.3 & 36.3 & 12.8 & 8.0 & 5.0 \\
\hline 4 & 461.2 & 48.0 & 23.1 & 7.6 & 5.0 \\
\hline 8 & 439.4 & 42.6 & 23.1 & 8.8 & 5.0 \\
\hline \multirow[t]{2}{*}{ Significance } & ${ }^{*} \mathrm{~L}$ & NS & ${ }^{*} \mathrm{~L}, \mathrm{Q}$ & NS & NS \\
\hline & \multicolumn{5}{|c|}{ Selection 535} \\
\hline 0 & 272.8 & 23.0 & 18.3 & 2.8 & 4.0 \\
\hline 4 & 383.8 & 32.3 & 21.8 & 4.2 & 3.4 \\
\hline 8 & 350.3 & 31.6 & 24.7 & 1.8 & 2.6 \\
\hline Significance & NS & NS & NS & NS & ${ }^{*} \mathrm{~L}$ \\
\hline
\end{tabular}

${ }^{2}$ Number of berries lost per cluster following $\approx 5 \mathrm{sec}$ of agitation immediately after removal from storage.

${ }^{y}$ Based on a scale where $1=0 \%-20 \% ; 2=2 \%-39 \% ; 3=40 \%-$ $59 \% ; 4=60 \%-79 \%$; and $5=80 \%-100 \%$ of the rachis displaying desiccation.

${ }_{\mathrm{s}, *, * *}$ Nonsignificant or significant at $P \leq 0.05$ or 0.01 , respectively. $\mathrm{L}=$ linear, $\mathrm{Q}=$ quadratic.

combinations (data not shown). In general, Selection 535 displayed considerable turgor loss in the berries, extensive rachis desiccation, and many moldly berries following $\approx 90$ days of storage. Total weight loss in storage averaged $30 \%$ for Selection 535 .

Expt. 3. Thidiazuron linearly increased cluster weight and berry weight in either fresh or stored clusters of the four varieties (Table 5). In some instances, the effects of thidiazuron were not significant, but the tendency in all cases was towards a linear increase in cluster and berry weight. Berries per cluster was not affected, nor was rachis length (data not shown). Inclement weather at bloom in 1990 caused uneven set in 'Simone', along with unusually low berry weight. Fruit composition was, for the most part, adversely affected by thidiazuron, with several significant linear decreases in degrees Brix and $\mathrm{pH}$ with increasing concentration (Table 5). TA was increased in Selections 495 and 535 , but was decreased by thidiazuron in 'Sovereign Coronation' and 'Simone'.

Several features of the post-storage condition of the clusters were affected by thidiazuron. Although weight loss in storage ranged from $7 \%$ for 'Sovereign Coronation' to 10 ' \% for Selection 495 and 'Simone', weight of clusters after storage still displayed an increasing linear trend with increased thidiazuron concentration (Table 6). Weight loss and rachis weight increased linearly as well in the two blue-skinned varieties, while Selection 495 also displayed a mainly quadratic rachis weight increase in response to increasing thidiazuron concentration. Clusters stored much better in 1990 than in 1989, presumably because of the use of $\mathrm{SO}_{2}$ pad and polyethylene covering; shatter increased in 'Simone' with increased thidiazuron, but rachis drying tended to decrease with increasing thidiazuron concentration. Berry shrivel was minimal to nil in all selections, and the occurrence of moldly berries and shatter after $48 \mathrm{~h}$ at $20 \mathrm{C}$ was negligible. Under the conditions used in 1990, Selection 495 showed the least potential for long-term storage and Selection 535 the most.

\section{Discussion}

Evidence presented here indicates that the substituted phenylurea compounds CPPU and thidiazuron may be used to increase cluster weight and berry size in several seedless grape varieties. This confirms results of Nickell (1985; 1986a, 1986b), who reported increased berry weight of 'Thompson Seedless', 'Flame', and 'Perlette' when treated with CPPU. Unlike Nickell's work, our results showed no beneficial or synergistic effects of GA when used in combination with CPPU.

Also contrary to Nickell's research, the present study clearly shows a tendency for CPPU to delay fruit maturity, resulting in lower degrees Brix and $\mathrm{pH}$, higher $\mathrm{TA}$, and, in blue-skinned varieties, lighter skin and juice color and lower juice anthocyanins. This delay is probably to be expected since compounds with cytokinin-like activity are known to slow senescence, of which fruit ripening is a specialized form. This effect may be a viticultural concern for late-season varieties, or those for which an early market is crucial. Ogata et al. (1988) also showed a tendency for CPPU to delay fruit maturity in 'Kyoho' grape.

This is the first report on the effects of thidiazuron on fruit size, fruit composition, and storage potential of grapes or any other fruit crop. In general, its effects appear to be equal to, if not better than, those of CPPU. Again, the apparent delaying of fruit maturity by thidiazuron could have negative consequences, depending on the variety and the region in which it is grown.

The impact of these two compounds upon storability of these seedless table grapes was largely negligible. Selection 535 showed some positive response to thidiazuron in terms of reduced rachis drying and berry shrivel, but the other selections displayed little or no beneficial effects. Although average weight loss per cluster increased with thidiazuron level in 1990, percentage weight loss did not vary with concentration.

Future research with these chemicals needs to focus on concentrations that will prove consistently effective when applied as whole vine sprays. In these experiments, cluster dips as low as $1 \mathrm{mg} \bullet \operatorname{liter}^{-1}$ of CPPU and thidiazuron contributed to significant increases in berry and cluster weight. However, according to Nickell (1985), foliar sprays would require $\approx 40 \%$ more chemical to achieve the same results as cluster dips. At the rates used in these experiments, this would extrapolate to spray levels of 2 to $17 \mathrm{mg} \cdot$ liter $^{-1}$. Whether these spray levels will be as effective as cluster dips for the increase of berry size in seedless grapes needs to be determined.

\section{Literature Cited}

British Columbia Ministry of Agriculture and Fisheries. 1987. Grape production guide. Victoria, B.C Denby, L.G. 1977. 'Sovereign Coronation' grape. HortScience 12:512.

wahori, S., S. Tominaga, and T. Yamasaki. 1988. Stimulation of fruit growth of kiwifruit, Actinidia chinesis Planch., by N-(2-chloro-4-pyridl)-N'-phenylurea, a diphenylurea-derivative cytokinin. Scientia Hort. 35:10915.

Métivier, R.P., F.J. Francis, and F.M. Clydesdale. 1980. Solvent extraction of anthocyanins from grape pomace. J. Food Sci. 45:1099-1101.

Morris, J. R., R.G. Butz, and L.C. Nickell. 1986. The effects of a new cytokinin, CN-11-3183, and GA on the yield and quality of Concord and Reliance grapes. 37th Annu. Mtg., Amer. Soc. Enol. Vitic., Anaheim, Calif. (Abstr.)

ickell, L.C. 1985. New plant growth regulator increases grape size. Proc. Plant Growth Regulat. Soc. Amer.

Nickell, L.O. 1986a. The effects of N-(2-chloro-4-pyidyl)-N'-phenylurea and the 3-chloro-benzyl ester of dicamba on

the growth and sugar content of grapes. Acta Hort. 179:805-806.
Nickell, L.G. 1986b. Effects of $\mathrm{N}$-(2-chloro-4-pyridyl) $-\mathrm{N}^{\prime}$-phenylurea on grapes and other crops. Proc. Plant Growth Regulat. Soc. Amer. 13:236-241.

Ogata, R., T. Saito, and K. Oshima. 1988. Effect of N-phenvl-N'-(4-pyrridyl) urea (4-PU) on fruit size: apple, japanese pear, grapevine, and kiwi fruit. Acta Hort. 239:395-398.

Reynolds, A.G., L.G. Denby, and M.J. Bouthillier. 1989. 'Simone' grape. HortScience 24:866-867.
Weaver, R.J., J. Van Overbeek, and R.M. Pool. 1966. Effect of kinins on fruit set and development in Viti. vinifera. Hilgardia 37:181-201. 